\title{
Анализ структуры и проводимости изогнутых цепочек углерода, полученных методом импульсного плазменного осаждения на различных металлических подложках
}

\author{
(С) И.П. Иваненко ${ }^{1}$, С.В. Краснощеков ${ }^{2}$, А.В. Павликов ${ }^{1}$ \\ ${ }^{1}$ Московский государственный университет им. М.В. Ломоносова (физический фракультет), \\ 119991 Москва, Россия \\ ${ }^{2}$ Московский государственный университет им. М.В. Ломоносова (химический фракультет), \\ 119991 Москва, Россия \\ E-mail: ivanenko@physics.msu.ru
}

(Получена 8 июня 2017 г. Принята к печати 19 июня 2017 г.)

Пленки линейно-цепочечного углерода толщиной порядка 100 нм изучались методом туннельной спектроскопии. Обнаружена осциллирующая зависимость дифференциальной проводимости исследуемых структур. Полученные результаты интерпретировались с использованием модели формирования волн зарядовой плотности в регулярных изгибах структуры углеродных цепочек. Получены спектры комбинационного рассеяния пленок. Проведено теоретическое соотнесение моделированных спектров гармонических колебаний полиинов $(-\mathrm{C} \equiv \mathrm{C}-)_{n}$ и кумуленов $(=\mathrm{C}=)_{n}$ углеродных пленок.

DOI: $10.21883 /$ FTP.2018.07.46050.8660

\section{1. Введение}

Одной из наиболее перспективных форм углеродных материалов является линейно-цепочечный углерод (ЛЦУ) [1]. В этой работе изучались углеродные пленки, полученные с использованием низкотемпературной импульсной ионно-стимулированной плазменной технологии [2]. ЛЦУ состоит из цепочек атомов углерода, ориентированных перпендикулярно поверхности подложки. Структура и электрические свойства этих пленок изучались ранее в работах [3-5]. В последние годы проявляется повышенный интерес к пленкам ЛЦУ [5].

Цель данной работы - изучение транспортных свойств ЛЦУ с использованием метода туннельной спектроскопии (ТС), анализ их молекулярной структуры путем сравнения экспериментальных и расчетных спектров комбинационного рассеяния света (КРС) и изучение корреляции между свойствами материала подложки и осажденной структуры. Исследована зависимость транспортных свойств конденсированных пленок ЛЦУ на различных материалах подложки. Были выбраны следующие металлы подложки: $\mathrm{Cu}, \mathrm{Mo}, \mathrm{W}$ и $\mathrm{Al}$. Средняя толщина конденсированных пленок на подложке составляла около $100 \mathrm{Hм}$.

Исследуя транспортные процессы в гетероструктурах (металлическая подложка-ЛЦУ) с помощью метода ТС, получаем информацию об их электрофизических свойствах, что подтверждается другими исследованиями [611]. Этот метод основан на явлении туннелирования электронов через потенциальный барьер между металлическим зондом, состоящим из сплава Pt и Ir, и поверхностью образца в приложенном электрическом поле. Величина туннельного тока в основном определяется коэффициентом пропускания барьера (расстоянием до поверхности и приложенным напряжением). Кроме того, на туннельный ток влияет форма плотности состояний, как на кончике микроскопа, так и в пленке, а также на подвижность электронов в слое проводимости пленки $[12,13]$.

\section{2. Эксперимент}

Исследования проводимости полученных гетероструктур проводились с использованием микроскопа FemtoScan [14] в режиме туннельной спектроскопии. Вольт-амперные характеристики (BAX) измерялись при фиксированном положении зонда в одной точке над поверхностью образца. Вычислены дифференциальные проводимости структур. На рис. 1 представлена типичная дифференциальная ВАХ для пленок.

Плотность туннельного тока может быть описана формулой Симмонса [12]. Для интерпретации экспе-

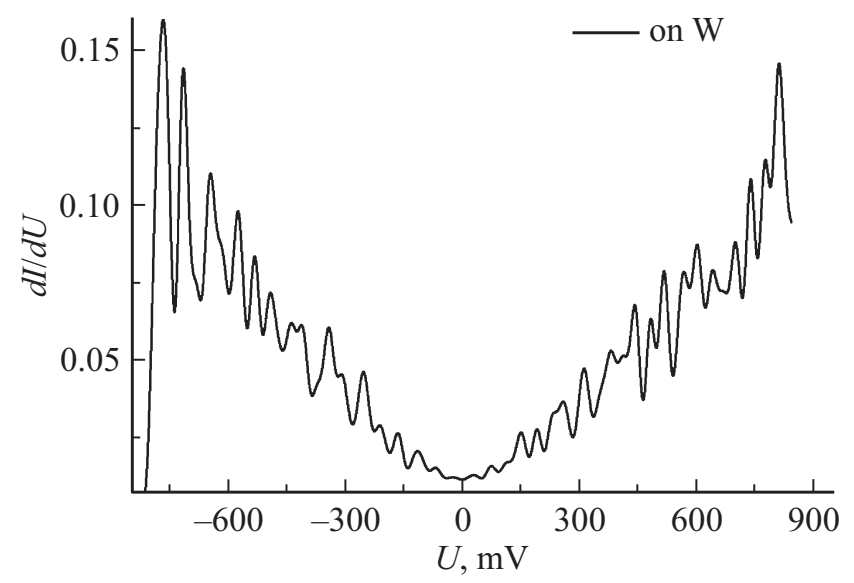

Рис. 1. Типичный график туннельных осцилляций проводимости для пленки, нанесенной на вольфрам. 
риментальных данных использование общей формулы затруднительно. Поэтому использовалось одно из трех приближений $[12,13]$. Наши условия эксперимента соответствуют случаю, когда приложенное напряжение сравнимо с работой выхода поверхности.

Эффекты осцилляций дифференциальной проводимости, обнаруженные при комнатной температуре, имеют особенности, обусловленные специфической структурой углерода. Для всех металлических подложек, используемых в эксперименте, дифференциальные осцилляции проводимости наблюдались с примерно одинаковым периодом для каждой структуры, который рассчитывается путем усреднения всех пиков. Период колебаний в несколько раз больше тепловой энергии при $300 \mathrm{~K}$. Подобные колебания дифференциальной проводимости в углеродных и полупроводниковых структурах наблюдались в предыдущих исследованиях [6-9].

В нашем случае предполагалось образование одномерных волн зарядовой плотности (ВЗП) вдоль углеродной цепочки. Период стоячей волны в ЛЦУ должен коррелировать с длиной линейного фрагмента, образуя суперструктуру. Очевидно, амплитуда волновой функции электропроводности при изгибе цепочки должна быть равна нулю. Для подложек $\mathrm{Cu}, \mathrm{W}, \mathrm{Mo}$ и $\mathrm{Al}$ было получено среднее число атомов углерода $n$ в цепи, равное $28,22,18$ и 12 соответственно, рассчитанное по формуле

$$
n=\left(\frac{12.25}{2 \sqrt{E}}\right) / 1.3
$$

Среднее расстояние между атомами углерода в цепочке принималось равным 0.130 нм, а длина волны рассчитывалась на основе волны де Бройля. Ранее Тиквинским и соавторами были получены молекулы с 44 атомами углерода [15].

\section{3. Метод расчета}

Для идентификации типов углеродных связей в синтезированной структуре использовались комбинационная спектроскопия и моделирование возможных структур (см. рис. 2). Как показано далее, модель без изгибов не объясняет спектры комбинационного рассеяния. Расчет гармонических колебаний молекул, состоящих из углеродных цепочек кумуленового $(=\mathrm{C}=\mathrm{C}=)$ и полиинового типа $(-\mathrm{C} \equiv \mathrm{C}-)$, проводился с использованием их основной структуры без изгибов между линейными фрагментами или с единственным изгибом между линейными фрагментами. Терминальные группы были смоделированы атомами водорода, основываясь на данных работ [16-19].

Оптимизация геометрических структур, расчет матрицы вторых производных электронной энергии и производных компонентов электрооптических свойств проводились с использованием программного пакета Gaussian 9.0 [20]. Дальнейший расчет нормальных колебаний и моделирование спектров комбинационного рас-

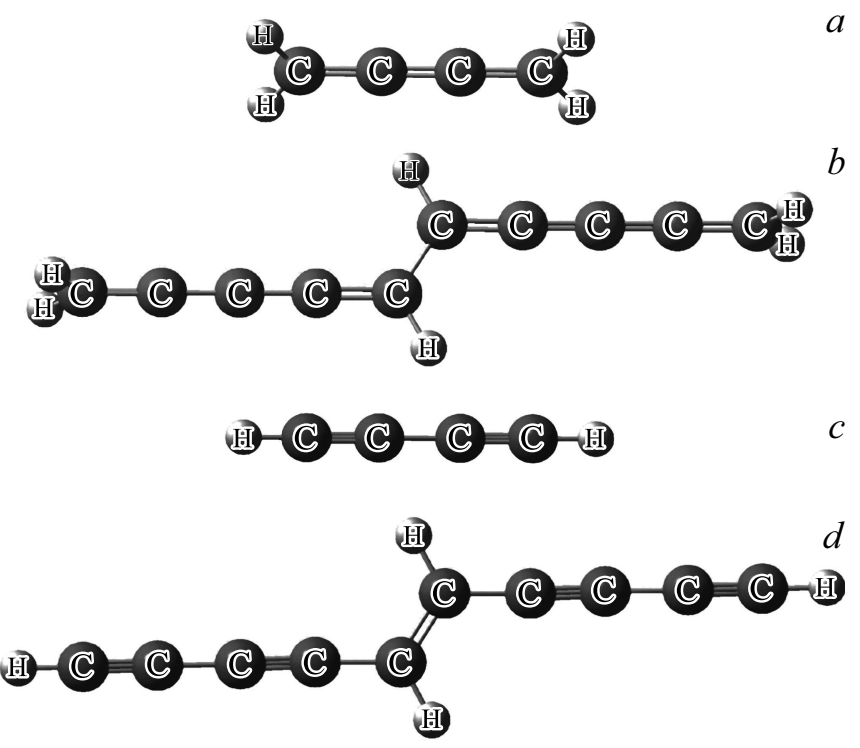

Рис. 2. Рассчитанные модели: $a$ - одиночный кумулен, состоящий из четырех атомов углерода, $b$ - двойной кумулен, состоящий из десяти атомов углерода, $c$ - одиночный полиин, состоящий из четырех атомов углерода, и $d-$ состоящий из двойного полиина с десятью атомами углерода. Использование одиночной цепочки означает использование одиночной линейной цепочки атомов углерода, а использование двойной цепочки означает, что между линейными компонентами возникает изгиб.

сеяния света проводились с использованием программы $\mathrm{ANCO}$, согласно проведенным исследованиям [21-24].

Расчет оптимизированных геометрических структур и гармонических силовых полей выполнялся с использованием квантово-механического метода Меллера- Плессе 2-го порядка (MP2) с базисным набором aug-cc-pVTZ. Достаточность модели была продемонстрирована в работе [25].

Для изучения влияния числа атомов углерода на предсказанные колебательные моды был выбран следующий набор молекул: (ps) одиночные полиины (4, 6 и 8 атомов углерода), (pd) двойной полиин (с изгибом) (3 и 5 атомов в молекуле), (cs) одиночный кумулен $(3,5$ и 6 атомов углерода) и (cd) двойной кумулен (4 и 5 атомов). Рассчитанные спектры для всех типов молекул представлены на рис. 3.

Размер и сложность исследуемых молекул, разнообразие их структур, наличие карбидной связи с подложкой $(\mathrm{Cu}, \mathrm{Mo}, \mathrm{W}$ и $\mathrm{Al})$ приводят к довольно сложным экспериментальным спектрам.

На рис. 4 представлены экспериментальные спектры КРС. Для регистрации спектров КРС нами использовался микрорамановский спектрометр Horiba Jobin Yvon HR800. Возбуждение спектров КРС проводилось с помощью лазера с длиной волны 633 нм. Как легко видеть, наилучшее согласие между теорией и экспериментом наблюдается для длинноцепочечных (pd) и (cd) типов. 


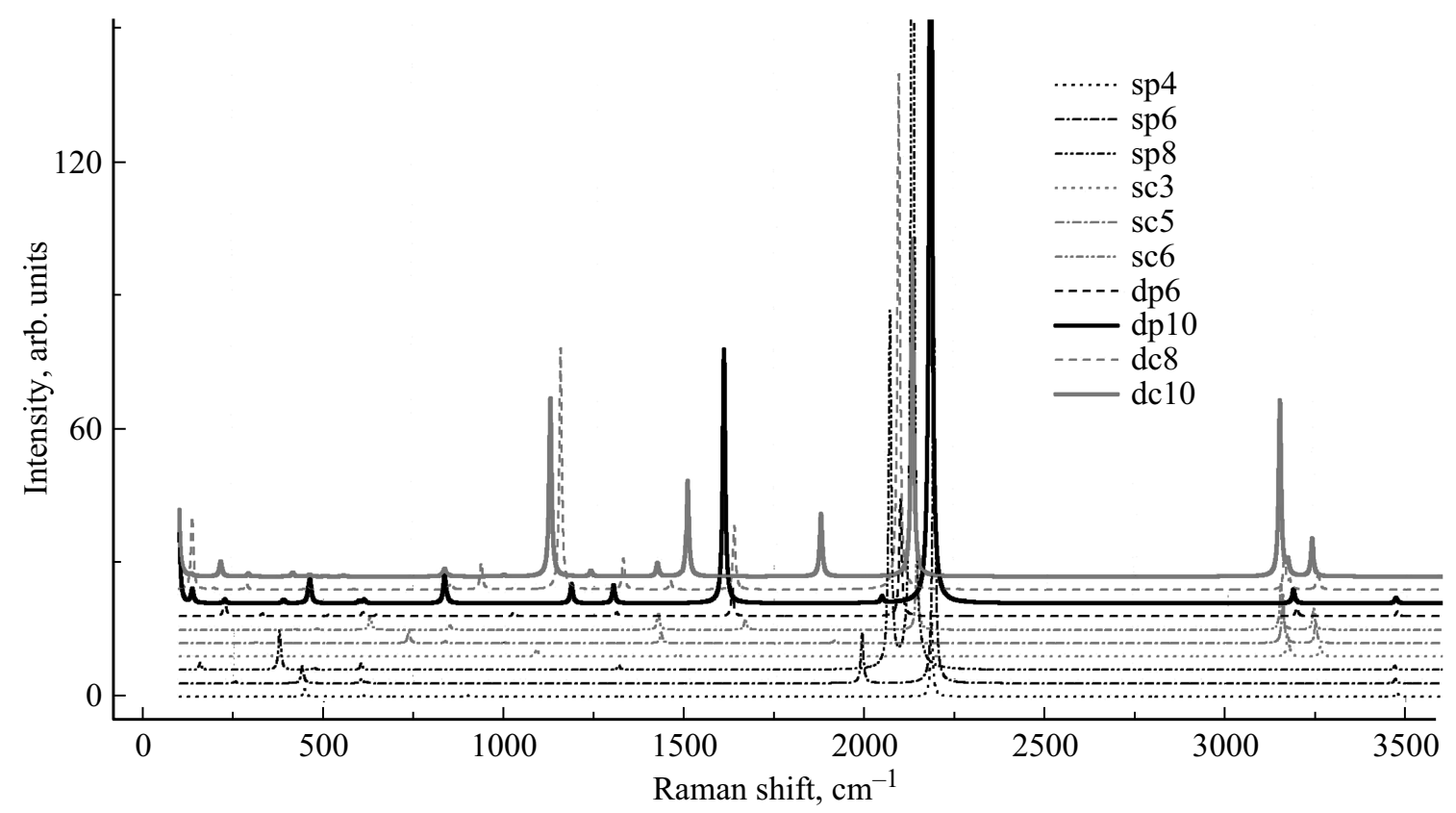

Рис. 3. Расчетные спектры комбинационного рассеяния молекул. sp - одиночный полиин, sc - одиночный кумулен, dp - двойной полиин, dc - двойной кумулен, последующее число представляет собой число атомов углерода в структурах.
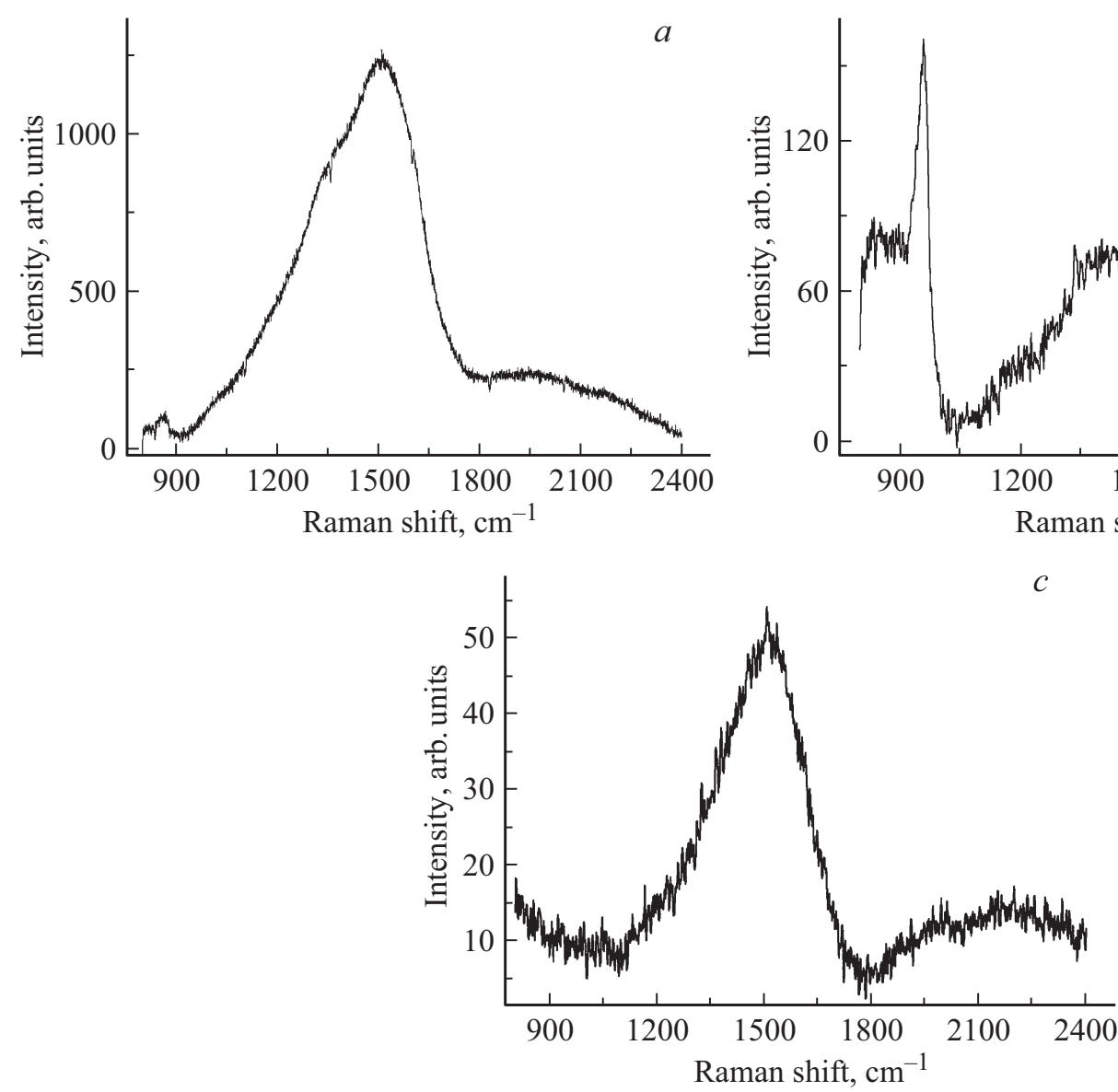

$b$

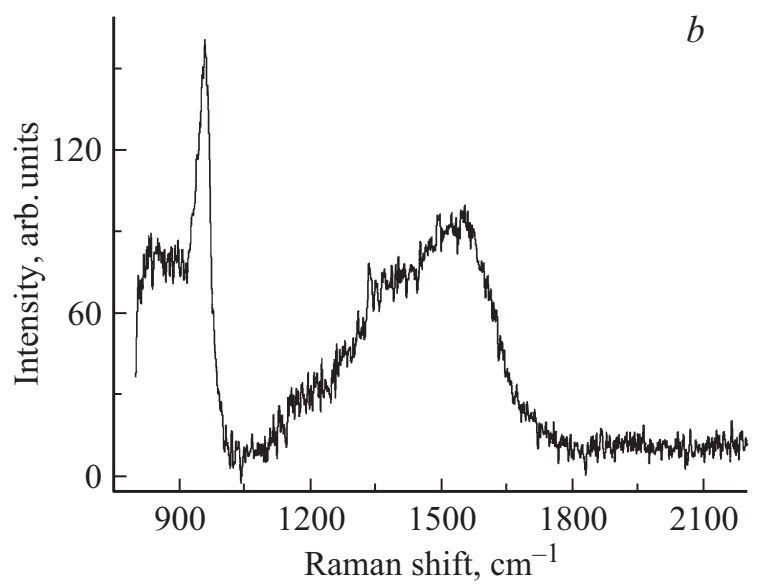


Действительно, в экспериментальном спектре КРС наблюдается широкая полоса с максимумом интенсивности $\left(\sim 1600 \mathrm{~cm}^{-1}\right)$, соответствующим частоте колебаний двойной связи на изгибе между линейными фрагментами цепочки, который известен как $G$-пик [26,27].

Расчеты показывают, что полосы в области $1000-1700 \mathrm{~cm}^{-1}$ указывают на присутствие молекул, принадлежащих структурам с наличием изгибов между цепочками $(\mathrm{cd} 8, \operatorname{cd} 10, \operatorname{pd} 6$ и $\mathrm{pd} 10)$, что не наблюдалось в моделях без изгиба или в предыдущем исследовании [26]. Но также появляется низкочастотная область $400-980 \mathrm{~cm}^{-1}$, которая ранее не была описана в литературе [5]. К низкочастотной области относятся колебания атомов углерода, которые распространяются параллельно подложке (вне плоскости).

Значения колебательных частот, связанных с изгибом цепочки для модели рd10, составляют 1304 и $1229 \mathrm{~cm}^{-1}$, что отвечает $\delta(\mathrm{C}=\mathrm{C}-\mathrm{H})$ def. и $1609 \mathrm{~cm}^{-1}$ до $v(\mathrm{C}=\mathrm{C})$ для изгиба; для модели рd6: 1313 и $1282 \mathrm{~cm}^{-1}$, ответственных за $\delta(\mathrm{C}=\mathrm{C}-\mathrm{H})$ def. и $1634 \mathrm{~cm}^{-1}$ к $v(\mathrm{C}=\mathrm{C})$ для изгиба; для модели dc8: $1158 \mathrm{~cm}^{-1}$ к $\delta(\mathrm{C}=\mathrm{C}-\mathrm{H})$ def. и $v(\mathrm{C}=\mathrm{C})$ для изгиба, $1332 \mathrm{~cm}^{-1}$ к $\delta(\mathrm{C}=\mathrm{C}-\mathrm{H})$ def. и $v(\mathrm{C}=\mathrm{C})$ str. около изгиба, $1639 \mathrm{~cm}^{-1} \delta(\mathrm{C}=\mathrm{C}-\mathrm{H})$ def., $v(\mathrm{C}=\mathrm{C})$ str. и $\delta(\mathrm{C}=\mathrm{C}-\mathrm{H})$ def. в конце цепочки; для модели $\mathrm{dc} 10: 1128$, 1241 и $1509 \mathrm{~cm}^{-1} \delta(\mathrm{C}=\mathrm{C}-\mathrm{H})$ def. и $v(\mathrm{C}=\mathrm{C})$ str. вблизи изгиба, $1425 \mathrm{~cm}^{-1} \delta(\mathrm{C}=\mathrm{C}-\mathrm{H})$ def. с $\delta(\mathrm{C}=\mathrm{C}-\mathrm{H})$ def. на конце цепочки. Особенности спектрального диапазона $1300-1600 \mathrm{~cm}^{-1}$ связаны с наличием $\mathrm{sp}^{2}$-связей [28].

Как видно из таблицы, полосы при 1129 и $1158 \mathrm{~cm}^{-1}$ связаны с колебаниями связи $\mathrm{C}-\mathrm{C}$ и относятся к структурам pd10 и $\mathrm{pd} 8$ соответственно. Они близки к полосам при 1140 и $1470 \mathrm{~cm}^{-1}$, что характерно для размеров нанокристаллов алмаза менее 2 нм [29]. Полосы с частотами 1609 и $1634 \mathrm{~cm}^{-1}$ связаны с колебаниями двойных связей $\mathrm{C}=\mathrm{C}$ и относятся к структурам $\mathrm{cd} 10$ и cd6 соответственно.

Одиночная связь $\mathrm{C}-\mathrm{C}$ характеризуется частотами в области $1188 \mathrm{~cm}^{-1}$ для модели pd10 и $1319 \mathrm{~cm}^{-1}$ (этот максимум часто называют $D$ пик для модели $\mathrm{ps} 8$ ). Положение этой полосы близко к частоте $1330 \mathrm{~cm}^{-1}$, что соответствует „нормальному“ алмазу со значительно большими размерами кристаллитов [28]. Двойная связь $\mathrm{C}=\mathrm{C}$ характеризуется частотами 2132 и $1879 \mathrm{~cm}^{-1}$ для модели cd10. Для модели $\mathrm{cd} 8$ эти частоты смещаются к 2095 и $1639 \mathrm{~cm}^{-1}$. В случае линейной молекулы для этой связи полосы имеют меньшую интенсивность и положения этих полос соответствуют частотам 2145 и $1670 \mathrm{~cm}^{-1}$ для модели cs6, тогда как для модели cs5 частоты равны 2187 и $1916 \mathrm{~cm}^{-1}$, а для модели $\mathrm{pd} 10-$ $1609 \mathrm{~cm}^{-1}$. Связь $\mathrm{C} \equiv \mathrm{C}$ характеризуется частотами 2182 и $2047 \mathrm{~cm}^{-1}$ для модели $\mathrm{pd} 10$, а уменьшение размера молекулы до 6 атомов (модель рd6) дает значение частот 2100 и $1634 \mathrm{~cm}^{-1}$ соответственно. В случае линейной молекулы соответствующая мода имеет пик при $2186 \mathrm{~cm}^{-1} \mathrm{c}$ низкой интенсивностью. Полоса между 1800 и $2200 \mathrm{~cm}^{-1}$, так называемая $C$-полоса, связана с режимами растяжения $s p$-гибридизованного углерода.
$C$-полоса состоит из основного пика около $2100 \mathrm{~cm}^{-1}$ $C 2$ и плеча с более низким пиком частоты $C 1$ около $1980 \mathrm{~cm}^{-1}$ [30].

Область спектра с характерными колебаниями тройных связей $\mathrm{C} \equiv \mathrm{C}$ также содержит частоты для одиночных связей: 2133 и $2070 \mathrm{~cm}^{-1}$ (в модели ps8). Аналогичный случай наблюдается для области частот связи $\mathrm{C}-\mathrm{C}$, которая совпадает с частотами для изгиба $\mathrm{C}-\mathrm{C}-\mathrm{H}$ на изгибах цепочки, создавая частоту $1157 \mathrm{~cm}^{-1}$.

Экспериментальные спектры были разложены на составляющие, описываемые гауссовой формой, как было предложено в работах [31-33]. Сравнивая моделированные спектры с наблюдаемыми для пленок с различными материалами подложки, можно сделать вывод, что пленка на $\mathrm{Cu}$, по-видимому, принадлежит модели pd10, что соответствует исследованиям в работе [34] с включениями моделей cs3 и pd6, пленка на Мо принадлежит $\operatorname{cd} 8$ с небольшими долями $\mathrm{pd} 10$ и $\operatorname{cd} 10$, a пленка на $\mathrm{W}$ принадлежит $\mathrm{cd} 10$ с включением $\mathrm{cd} 8$ и пленка на $\mathrm{Al}$ соответствует структуре $\mathrm{cd} 10$ с включениями pd6, cs6 и cd8. Это может быть связано с примесями в металле. Метод синтеза, используемый для пленки, включает ионную бомбардировку подложки с последующим распылением металла подложки и возможной диффузией последнего вдоль цепочки. Таким образом, можно предположить, что атомы подложки могут служить примесями, связанными с изгибами в ЛЦУ.

В спектрах всех пленок наблюдается максимум с частотой, близкой к $1050 \mathrm{~cm}^{-1}$, который называют $T$-пиком, как наблюдалось в работе [35], но он был обнаружен только при возбуждении в УФ области. Мы можем наблюдать полосу с частотой $\sim 1470 \mathrm{~cm}^{-1}$, которая в литературных данных описывалась как характерная только для пленок, состоящих из углеродных шаров [36], или для трансполимера (СС-мода растяжения), частоты 1078 и $1458 \mathrm{~cm}^{-1}$ [37]. Для аморфного углерода характерна широкая полоса с центром $1480 \mathrm{~cm}^{-1}$ [38]. Три максимума 996, 1116 и $1498 \mathrm{~cm}^{-1}$ также появляются в спектре аморфного углерода или могут быть отнесены к структуре кумуленового карбина, стабилизированного дисперсными атомами $\mathrm{Cu}$ от подложки [39], а полосы в области $2150 \mathrm{~cm}^{-1}$ связаны с полииновой структурой карбинов [40,41]. Полоса с частотой $1250 \mathrm{~cm}^{-1}$ была обнаружена в углероде типа графон [42]. Очень полезными являются изменения диаграммы спектров комбинационного рассеяния аморфного углерода, представленные в работе [28]. Широкая форма полос комбинационного рассеяния света может быть вызвана случайным сдвигом следующего звена в длинной цепочке.

Были также рассчитаны углы изгиба пленок ЛЦУ для $\mathrm{dc} 8, \mathrm{dc} 10, \mathrm{dc} 12: 122.00,122.190,122.720$ и для dp6 и dp10: 122.560 и 122.480 , что согласуется с данными работы [17]. Разница заключается в используемой модели самой цепочки с учетом BLA. 
Экспериментальные значения частот колебаний в спектрах КР для выделения подложек $\mathrm{Cu}, \mathrm{Mo}, \mathrm{W}$ и $\mathrm{Al}$ на основе гармонического анализа

\begin{tabular}{|c|c|c|c|c|c|}
\hline \multicolumn{4}{|c|}{$\begin{array}{c}\text { Эксперимент: подложка, } \\
\text { волновое число, интенсивность }\end{array}$} & \multirow{2}{*}{$\begin{array}{c}\text { Условное } \\
\text { обозначение }\end{array}$} & \multirow{2}{*}{$\begin{array}{c}\text { Вычисленная частота: тип молекулы, } \\
\text { частота }\left(\mathrm{cm}^{-1}\right) \text {, интенсивность }(\AA / a . m . u .), \\
\text { значение основной частоты }\left(v_{i}\right)\end{array}$} \\
\hline $\mathrm{Cu}$ & Mo & $\mathrm{W}$ & $\mathrm{Al}$ & & \\
\hline & $2140 \mathrm{~m}$ & $2171 w$ & $2226 \mathrm{vw}$ & $\begin{array}{l}v(\mathrm{C} \equiv \mathrm{C}) \text { str. } \\
v(\mathrm{C}=\mathrm{C}) \text { str. }\end{array}$ & $\begin{array}{c}\mathrm{dc} 10: v_{2}=2132(3470) ; \operatorname{sc} 5: v_{7}=2187(3) \\
\text { sc6: } v_{2}=2145(783) ; \mathrm{dp} 10: v_{2}=2182(10698) \\
\text { sp8: } v_{2}=2133(12992) ; \operatorname{sp6}: v_{2}=2192(3942) ; \\
\text { sp4: } v_{2}=2186(792)\end{array}$ \\
\hline \multirow[t]{4}{*}{$2070 \mathrm{~s}$} & 2011vw & $2002 \mathrm{~m}$ & $2077 w$ & $\begin{array}{l}v(\mathrm{C} \equiv \mathrm{C}) \text { str. } \\
v(\mathrm{C}=\mathrm{C}) \text { str. }\end{array}$ & $\begin{array}{c}\text { dc8: } v_{3}=2094(5211) ; \operatorname{sc} 3: v_{14}=2031(3) ; \\
\text { dp10: } v_{4}=2048(62) ; \text { dp6: } v_{3}=2099(1194) \\
\text { sp8: } v_{3}=2071(3542) ; \operatorname{sp6}: v_{3}=1993(486)\end{array}$ \\
\hline & $1953 \mathrm{vw}$ & & & $\begin{array}{l}v(\mathrm{C} \equiv \mathrm{C}) \text { str. } \\
v(\mathrm{C}=\mathrm{C}) \text { str. }\end{array}$ & sc5: $v_{2}=1916(27) ;$ sp6: $v_{3}=1993(486)$ \\
\hline & $1863 \mathrm{~m}$ & $1874 w$ & $1895 \mathrm{vw}$ & $\begin{array}{c}\delta(\mathrm{C}=\mathrm{C}-\mathrm{H}) \text { sciss. } \\
\quad v(\mathrm{C}=\mathrm{C}) \text { str. }\end{array}$ & $\mathrm{dc} 10: v_{3}=1878(568)$ \\
\hline & & & $1664 \mathrm{~s}$ & $\begin{array}{c}v(\mathrm{C}=\mathrm{C}) \text { str. } \\
v(\mathrm{C}-\mathrm{C}-\mathrm{H}) \text { str. }\end{array}$ & $\begin{array}{c}\text { dc8: } v_{4}=1639(504) ; \text { sc6: } v_{3}=1669(90) \\
\text { dp10: } v_{5}=1609(1972) ; \text { dp6: } v_{4}=1634(218)\end{array}$ \\
\hline \multirow[t]{2}{*}{$1494 \mathrm{vs}$} & $1547 \mathrm{~s}$ & $1506 \mathrm{vs}$ & $1507 \mathrm{vs}$ & $\begin{array}{c}\delta(\mathrm{C}=\mathrm{C}-\mathrm{H}) \text { sciss. } \\
v(\mathrm{C}=\mathrm{C}) \text { str. } \\
(\mathrm{C}-\mathrm{C}-\mathrm{H}) \text { str. }\end{array}$ & $\begin{array}{c}\text { dc10: } v_{4}=1509(693) ; \operatorname{sc} 3: v_{2}=1483(19) \\
\text { dp10: } v_{5}=1609(1972)\end{array}$ \\
\hline & & & & $\delta(\mathrm{C}=\mathrm{C}-\mathrm{H})$ sciss. & $\begin{array}{c}\operatorname{dc} 10: v_{5}=1426(92) ; \operatorname{dc} 8: v_{5}=1463(65) \\
\text { sc3: } v_{2}=1483(19) ; \operatorname{sc} 5: v_{3}=1436(75) \\
\text { sc6: } v_{4}=1428(124)\end{array}$ \\
\hline $1337 \mathrm{vs}$ & $1358 \mathrm{~s}$ & $1349 \mathrm{~m}$ & $1333 \mathrm{~s}$ & $\begin{array}{c}v(\mathrm{C}-\mathrm{C}) \text { str. } \\
\delta(\mathrm{C}=\mathrm{C}-\mathrm{H}) \text { sciss. } \\
\chi(\mathrm{C}-\mathrm{C}-\mathrm{H}) \text { rock. }\end{array}$ & $\begin{array}{c}\text { dc8: } v_{6}=1332(201) ; \operatorname{sc} 5: v_{9}=1310(1) \\
\text { dp10: } v_{6}=1304(115) ; \operatorname{dp6}: v_{5}=1313(27) \\
\text { sp8: } v_{4}=1318(30)\end{array}$ \\
\hline \multirow[t]{2}{*}{$1262 \mathrm{~s}$} & $1205 \mathrm{~m}$ & $1218 \mathrm{~m}$ & $1208 w$ & $\begin{array}{c}\delta(\mathrm{C}=\mathrm{C}-\mathrm{H}) \text { sciss. } \\
v(\mathrm{C}=\mathrm{C}) \text { str. }\end{array}$ & $\mathrm{dc} 10: v_{6}=1241(34) ; \mathrm{dp} 10: v_{7}=1187(109)$ \\
\hline & $1147 \mathrm{~m}$ & & & $\begin{array}{c}v(\mathrm{C}-\mathrm{C}) \text { str., } \\
v(\mathrm{C}=\mathrm{C}) \text { str., } \\
\delta(\mathrm{C}=\mathrm{C}-\mathrm{H}) \text { sciss., } \\
\chi(\mathrm{C}-\mathrm{C}-\mathrm{H}) \text { rock. }\end{array}$ & $\begin{array}{c}\operatorname{dc} 10: v_{7}=1129(968) ; \operatorname{dc} 8: v_{7}=1158(1345) ; \\
\quad \operatorname{dp} 10: v_{7}=1187(109) .\end{array}$ \\
\hline \multirow[t]{3}{*}{$1077 \mathrm{~m}$} & $1086 \mathrm{~m}$ & $1036 w$ & $1083 \mathrm{~m}$ & $\begin{array}{c}v(\mathrm{C}=\mathrm{C}) \text { str. } \\
\chi(\mathrm{C}=\mathrm{C}-\mathrm{H}) \text { rock. }\end{array}$ & $\begin{array}{l}\text { dc10: } v_{29}=1000(9) ; \mathrm{dc} 8: v_{8}=1027(8) \\
\text { sc3: } v_{3}=1092(46) ; \mathrm{dp6}: v_{6}=1028(24)\end{array}$ \\
\hline & & & & $\begin{array}{c}v(\mathrm{C}-\mathrm{C}) \text { str. } \\
v(\mathrm{C}=\mathrm{C}-\mathrm{H}) \text { sciss. }\end{array}$ & dc8: $v_{8}=1027(8) ;$ dp6: $v_{6}=1028(24)$ \\
\hline & $954 v s$ & & $987 \mathrm{vw}$ & $\begin{array}{c}v(\mathrm{C}=\mathrm{C}) \text { str., } \\
\chi(\mathrm{C}=\mathrm{C}-\mathrm{H}) \text { rock. }\end{array}$ & dc10: $v_{29}=1000(9) ; \operatorname{dc} 8: v_{9}=938(122)$ \\
\hline $827 \mathrm{v}$ & & & $858 w$ & $\begin{array}{c}\nu(\mathrm{C}-\mathrm{C}) \text { str. } \\
\delta(\mathrm{C}=\mathrm{C}-\mathrm{H}) \text { wag. } \\
\chi(\mathrm{C}-\mathrm{C}-\mathrm{H}) \text { rock. }\end{array}$ & $\begin{array}{c}\mathrm{dc} 10: v_{32}=847(5) ; \operatorname{sc} 5: v_{14}=837(4) ; \\
\text { sc6: } v_{14}=851(17) ; \operatorname{dp} 10: v_{8}=835(117) \\
\text { dp6: } v_{14}=850(14)\end{array}$ \\
\hline
\end{tabular}

Примечание. * Интенсивность полос: очень слабые - vw, слабые - w, средние - m, сильные - s, очень сильные - vs. ${ }^{* *}$ Описание режимов: $v$ - валентные, $\delta$ - деформационные, $\chi$ - вне плоскости.

Корреляция интенсивности полос в спектрах комбинационного рассеяния света с морфологией пленки позволяет сделать вывод о том, что увеличение интенсивности сигнала можно объяснить проявлением эффекта гигантского КРС. Согласно данным АСМ, поверхность является высокоразвитой, высота структур составляет 280-900 нм (см. рис. 5, $b$ ). Они представляют собой скопление атомов $\mathrm{Cu}$, покрытых углеродной пленкой.

Поверхности пленок, полученных на Al-подложках, также были исследованы методом АCM, который по- 
$a$

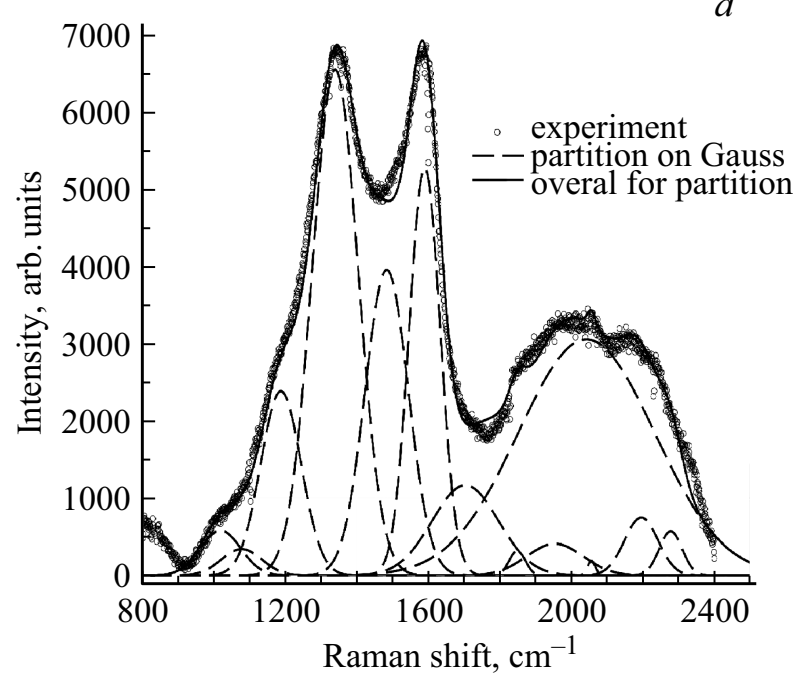

$b$

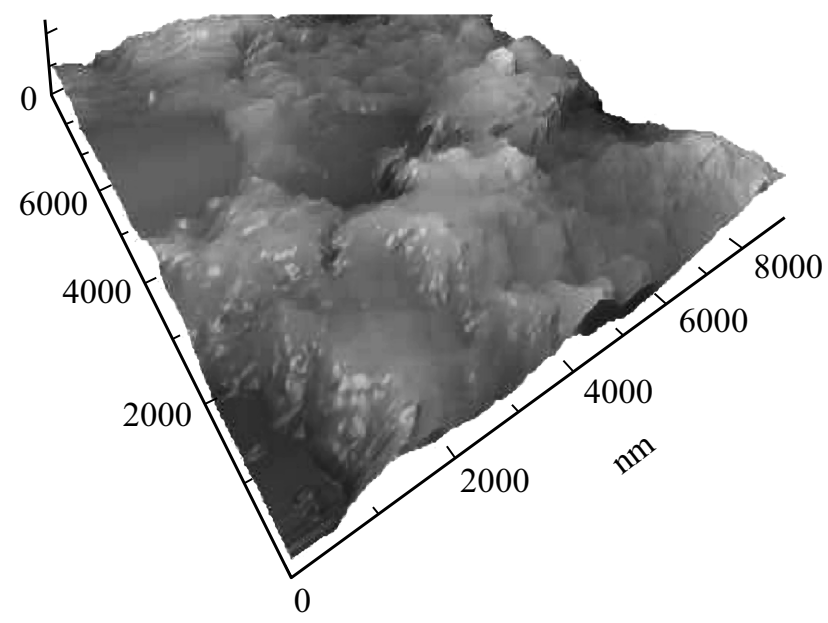

Pис. 5. Спектр пленки на подложке $\mathrm{Cu}$ с разложением на gauss-функции $(a)$, ACM-изображение поверхности пленки $(b)$.

казал следующие результаты: поверхность представляет собой кластеры с размерами от 170 до 250 нм. Сравнивая интенсивность сигнала КРС пленок, полученных на Al-подложке, с интенсивностью сигнала от пленок, выращенных на других подложках, можно заметить, что для данного случая интенсивность имеет промежуточное значение. Она меньше для пленок на $\mathrm{Cu}$ и больше для пленок на W и Мо. Так как для $\mathrm{Al}$ на используемой длине волны возбуждения (633 нм) не должно наблюдаться резонансного эффекта усиления, можно говорить о проявлении другого механизма усиления: так называемого „эффекта громоотвода“, не носящего резонансного характера.

Для пленок на подложках $\mathrm{W}$ и Мо наблюдалась менее развитая поверхность, что можно объяснить образованием соединений металлов с углеродом, так называемым карбидным слоем. Он имеет промежуточное положение между металлическим материалом подложки и углеродной пленкой. Этот слой блокирует образование диффузного слоя атомов металла в углеродной матрице. При комнатной температуре возможность образования такого промежуточного слоя обусловлена присутствием ионной стимуляции при синтезе пленок.

\section{4. Заключение}

Настоящее исследование связано с осаждением ЛЦУ на некоторые металлические подложки $(\mathrm{Cu}, \mathrm{Mo}, \mathrm{W}$ и Al) в одинаковых экспериментальных условиях. Для исследования их структуры и проводимости использовались КРС и туннельная спектроскопия. Для определения возможных структур пленок были смоделированы гармонические колебания молекул, содержащих углеродные цепочки с различными типами химических связей. Сравнение расчетных и экспериментальных спектров КРС приводит к выводу, что пленки, принадлежащие модели ЛЦУ, имеют изгибы цепочек. Длины линейных фрагментов рассчитывались на основе предположений, обнаруженных ранее [5]. Изучена зависимость структуры пленок от материала подложки.

Авторы выражают благодарность профессору М.Б. Гусевой, доц. В.В. Хвостову, доц. И.К. Гайнуллину, а также Р.С. Шуцкому за участие в обсуждениях результатов исследований и анализ полученных результатов.

\section{Список литературы}

[1] V.G. Babaev, M.B. Guseva, N.D. Novikov, V.V. Khvostov, P. Flood. Polyynes Synthesis, Properties and Application, ed. by F. Cataldo (CRC Press, Boca Raton, 2006).

[2] Patent US6.355.350.B1, Tetracarbon (2002).

[3] V.G. Babaev, M.B. Guseva, N.F. Savchenko, N.D. Novikov, V.V. Khvostov, P. Flad. J. Surface Investigation, X-ray, Synchrotron and Neutron Techniques, 3, 16 (2004).

[4] V.V. Khvostov, I.P. Ivanenko, O.A. Streletskiy, N.D. Novikov, V.G. Yakunin, N.F. Savchenko. JETP Lett., 97, 231 (2013),

[5] E.A. Buntov, A.F. Zatsepin, M.B. Guseva, Yu.S. Ponosov. Carbon, 117, 271 (2017).

[6] N.S. Maslova, S.I. Oreshkin,V.I. Panov, S.V. Savinov. JETP Lett., 67, 146 (1998).

[7] T.K. Zvonareva, V.I. Ivanov-Omskii, V.V. Rozanov, L.V. Sharonova. Semiconductors, 35, 1398 (2001).

[8] A.O. Golubok, O.M. Gorbenko, T.K. Zvonareva, S.A. Maslov, V.V. Rozanov, S.G. Yastrebov, V.I. Ivanov-Omskii. Semiconductors, 34, 217 (2000).

[9] N.S. Maslova, Yu.N. Moiseev, S.V. Savinov, R.G. Yusupov. JETP Lett., 58, 528 (1993).

[10] K.S. Nakayama, M.G. Alemany, T. Sugano, K. Ohmori, H. Kwak, J.R. Chelikowsky, J.H. Weaver. Phys. Rev. B, 73, 035330 (2006).

[11] Z. Klusek, P. Kowalczyk, P. Byszewski. Vacuum, 63, 145 (2001).

[12] J.G. Simmons. J. Appl. Phys., 34, 1793 (1963).

[13] J.G. Simmons. J. Appl. Phys., 34, 238 (1963). 
[14] Advanced technologies center RU, http://www.nanoscopy.net/en/

[15] W.A. Chalifoux, R.R. Tykwinski. Nat. Chem., 2, 967 (2010).

[16] A. Milani, M. Tommasini, V. Russo, A.L. Bassi, A. Lucotti, F. Cataldo, C.S. Casari. Beilstein J. Nanotechnol., 6, 480 (2015).

[17] J.G. Korobova, D.I. Bazhanov. JETP Lett., 93, 730 (2011).

[18] V.M. Melnichenko, A.M. Sladkov, J.N. Nikulin. J. Chemistry Successes (in Russian), 5, 736 (1982).

[19] C. Casiraghi, F. Piazza, A.C. Ferrari, D. Grambole, J. Robertson. Diamond Relat. Mater., 14, 1098 (2005).

[20] M.J. Frisch, G.W. Trucks, H.B. Schlegel et al. Gaussian'03, Revision B.03. (Pittsburgh, 2003).

[21] S.V. Krasnoshchekov, N.F. Stepanov. J. Phys. Chem., 82, 690 (2008).

[22] S.V. Krasnoshchekov, E.V. Isayeva, N.F. Stepanov. J. Phys. Chem. A, 116, 3691 (2012).

[23] S.V. Krasnoshchekov, N.C. Craig, P. Boopalachandran, J. Laane, N.F. Stepanov. J. Phys. Chem., 119, 10706 (2015).

[24] Y.N. Panchenko, S.V. Krasnoshchiokov, C.W. Bock. J. Comput. Chem., 9, 443 (1988).

[25] A. Daniel Boese, W. Klopper, J.M.L. Martin. Int. J. Quant. Chem., 104, 830 (2005).

[26] A.C. Ferrari, J. Robertson. Phys. Rev. B, 64, 075414 (2001).

[27] A. Milani, M. Tommasini, V. Russo, A. Li Bassi, A. Lucotti, F. Cataldo, S. Carlo. Beiltein J. Nanotechnol., 6, 4802015.

[28] A.C. Ferrari, J. Robertson. Phys. Rev. B, 61, 14095 (2000).

[29] A.N. Obraszov, A.P. Volkov I.Yu. Pavlovsky. JETP Lett., 68, 56 (1998).

[30] C.S. Casari, A. Li Bassi, A. Baserga, L. Ravagnan, P. Piseri, C. Lenardi, M. Tommasini, A. Milani, D. Fazzi, C.E. Bottani, P. Milani. Phys. Rev. B, 77, 195444 (2008).

[31] M. Rybachuka, J.M. Bellb. Carbon, 47, 2481 (2009).

[32] E. Cinquanta, L. Ravagnan, I. Eligio Castelli, F. Cataldo, N. Manini, G. Onida, P. Milani. J. Chem. Phys., 135, 194501 (2011).

[33] P.K. Chu, L. Li. Mater. Chem. Phys., 96, 253 (2006).

[34] J.A. Lenz, C.A. Perottoni, N.M. Balzaretti, J.A.H. da Jornada. J. Appl. Phys., 89, 8284 (2001).

[35] A.C. Ferrari, J. Robertson. Phys. Rev. B, 64, 075414 (2001).

[36] Sh. Li, G. Ji, Zh. Huang, F. Zhang, Y. Du. Carbon, 45, 2946 (2007).

[37] H. Kuzmany, P. Knoll. Springer Ser. Sol. St. Sci., 63, 114 (1985).

[38] M. Chhowalla, A.C. Ferrari, J. Roberston, G.A.J. Amaratunga. Appl. Phys. Lett., 76, 1419 (2000).

[39] F. Cataldo, D. Capitani. Mater. Chem. Phys., 59, 225 (1999).

[40] J.A. Lenz, C.A. Perottoni, N.M. Balzaretti, J.A.H. da Jornada. J. Appl. Phys., 89, 8284 (2001).

[41] L. Kavan, J. Hlavaty, J. Kastner, H. Kuzmany. Carbon, 33, 1321 (1995).

[42] A.V. Baranov, A.N. Bekhterev, Y.S. Bobovich, V.I. Petrov. Opt. Spectrosc. (USSR), 62, 613 (1987).

\section{Analysis of the structure and conductivity of kinked carbon chains obtained by pulsed plasma deposition on various metal substrate}

\author{
I.P. Ivanenko ${ }^{1}$, S.V. Krasnoshchekov ${ }^{2}$, A.V. Pavlikov ${ }^{1}$ \\ ${ }^{1}$ Lomonosov Moscow State University \\ (Faculty of Physics), \\ 119991 Moscow, Russia \\ ${ }^{2}$ Lomonosov Moscow State University \\ (Faculty of Chemistry), \\ 119991 Moscow, Russia
}

Abstract Films of linear-chain carbon with a thickness of order of $100 \mathrm{~nm}$ were studied with using method of tunneling spectroscopy. An oscillating dependence of the differential conductivity of the studied structures was detected. The results obtained were interpreted using a model of the formation of charge density waves in the kinks in the regular structure of carbon chains. Raman spectra of the films studied were obtained. The first detective theoretical modeling of spectra of harmonic vibrations of $(-\mathrm{C} \equiv \mathrm{C}-)_{n}$ polyine and $(=\mathrm{C}=)_{n}$ cumulene types of carbon films was carried out.

Редактор А.Н. Смирнов 